\title{
Mandenkan
}

MANDENIKAN Bulletin semestriel d'études linguistiques mandé

49 | 2013

Le maninka du Niokolo

\section{Groupes nominaux en fonction oblique}

Section 16

\section{Denis Creissels}

\section{(2) OpenEdition}

Journals

Édition électronique

URL : https://journals.openedition.org/mandenkan/612

DOI : $10.4000 /$ mandenkan. 612

ISSN : 2104-371X

Éditeur

Llacan UMR 8135 CNRS/Inalco

\section{Édition imprimée}

Date de publication : 1 juin 2013

Pagination : 99-108

ISSN : 0752-5443

\section{Référence électronique}

Denis Creissels, «Groupes nominaux en fonction oblique », Mandenkan [En ligne], 49 | 2013, mis en ligne le 25 avril 2014, consulté le 16 janvier 2023. URL : http://journals.openedition.org/mandenkan/ 612 ; DOI : https://doi.org/10.4000/mandenkan.612

Ce document a été généré automatiquement le 16 janvier 2023.

\section{(c) $)(1)(2)$}

Creative Commons - Attribution - Pas d'Utilisation Commerciale - Partage dans les Mêmes Conditions 4.0 International - CC BY-NC-SA 4.0

https://creativecommons.org/licenses/by-nc-sa/4.0/ 


\title{
Groupes nominaux en fonction oblique
}

\author{
Section 16
}

Denis Creissels

\subsection{Obliques non marqués}

1 L'emploi d'obliques non marqués s'observe dans les mêmes conditions que dans les autres variétés de mandingue, notamment avec les toponymes ainsi que les composés nominaux ayant pour tête dulaa 'endroit'.

\begin{tabular}{|l|l|l|l|l|l|l|}
\hline$(152)$ & a. & Máninkáa & běe & bo-ta & Mándiy & née. \\
\hline & & maninka.D & tous & sortir-ACPP & Mandé & FOC \\
\hline & & \multicolumn{3}{|l}{ 'Tous les Maninka viennent du Mandé.' } \\
\hline
\end{tabular}

\begin{tabular}{|c|c|c|c|c|}
\hline b. & $A$ & taya-ta & Kéeduðu & lée. \\
\hline & $3 S G$ & partir-ACPP & Kédougou & FOC \\
\hline & \multicolumn{4}{|c|}{ 'Il est parti à Kédougou.' } \\
\hline
\end{tabular}

\begin{tabular}{|c|c|c|c|c|}
\hline c. & Alu & ge & tazá & liiboodula!́a! \\
\hline & INCL & SUBJP & aller & miel-sortir-endroit.D \\
\hline & \multicolumn{4}{|c|}{ 'Partons chercher du miel!' } \\
\hline
\end{tabular}


Quelques noms communs se référant à des lieux ont la particularité de pouvoir signifier un repérage spatial à la forme nue sans se combiner à une postposition: banta 'extérieur', duí:yu 'sol', lo':itaani 'dispensaire', suu 'maison.

\begin{tabular}{|c|c|c|c|c|c|c|c|c|c|}
\hline (153) & a. & Kana:-a & tu & banta. & $=$ & Kana: & $t u$ & bant-0 & to. \\
\hline & & SUBJN & rester & extérieur & SUBJN & rester & extérieur-D & LOC & \\
\hline & & \multicolumn{8}{|c|}{ 'Ne reste pas dehors!' } \\
\hline
\end{tabular}

\begin{tabular}{|c|c|c|c|c|c|c|c|}
\hline b. & Ko & be & sanna, & $a$ & hakil-oiso & be & duíru. \\
\hline & oiseau.D & COPLOC & en_l'air & $3 S G$ & esprit-D & COPLOC & sol \\
\hline & \multicolumn{7}{|c|}{ 'L'oiseau est en l'air, mais son esprit est au sol.' } \\
\hline
\end{tabular}

Les noms de jours utilisés pour exprimer la datation ainsi que si-'ínaa 'fois' combiné à un numéral pour indiquer la répétition peuvent aussi être mentionnés à cette rubrique.

\begin{tabular}{|l|l|l|l|l|l|}
\hline (154) & a. & Tiba & me - e & ke & a \\
\hline & & couvrir & POTn & faire & jeudi-jour-D \\
\hline & \multicolumn{4}{|c|}{ 'On ne couvre pas les cases le jeudi.' } \\
\hline
\end{tabular}

\begin{tabular}{|c|c|c|c|c|c|c|}
\hline b. & $\eta$ & ges & $i$ & kilii & sicina & fuila. \\
\hline & $1 \mathrm{SG}$ & ACPP & $2 S G$ & appeler & fois & deux \\
\hline & \multicolumn{6}{|c|}{ 'Je t'ai appelé deux fois.' } \\
\hline
\end{tabular}

\subsection{Postpositions spécialisées}

4 L'inventaire des postpositions spécialisées inclut fee, kálama, kạ, kamma, la, ma, ti, to et yey - ex. (155) à (162). On peut attribuer un contenu sémantique précis à kálama ('au courant de'), kay ('sur'), kamma (motivatif), to (locatif, sans spécification d'une configuration spatiale particulière) et yey (bénéfactif). Par contre, de manière analogue à ce qui est décrit par Creissels et Sambou (2013) pour le mandinka, les postpositions fee, la, ma et ti expriment chacune une variété de significations qui ne se laisse pas réduire de façon évidente à un sens qu'on pourrait considérer comme leur valeur de base, et c'est la raison pour laquelle elles sont simplement glosées OBL. Comme cela a été observé dans d'autres parlers mandingues, on peut toutefois déceler dans les emplois de fer-ie une valeur originelle de proximité, dans les emplois de ma une valeur originelle de contact, et dans les emplois de la une valeur originelle de localisation. 
Mais la valeur de localisation de la tend à s'estomper du fait de la concurrence avec to, typiquement utilisée pour les repérages spatiaux concrets. L'emploi instrumental est l'emploi le plus productif de la. Enfin, ti a comme emplois productifs l'expression du fonctif ('en tant que', 'en qualité de') et de la comparaison, mais se trouve aussi en valeur comitative avec un nombre limité de verbes.

\begin{tabular}{|l|l|l|l|l|l|l|l|}
\hline (155) & a. & Basá & se & $i$ & mín & siisee & fee. \\
\hline & margouillat & POTP & REFL & boire & poule & OBL & \\
\hline & 'Le margouillat se désaltère aux dépens de la poule.' \\
\hline
\end{tabular}

\begin{tabular}{|l|l|l|l|l|l|}
\hline & b. & Móyo & té & $a$ & feee. \\
\hline & & personne & CoPN & $35 \mathrm{SG}$ & OBL \\
\hline & & \multicolumn{2}{|c|}{ 'Il n'a personne près de lui.' ou 'Personne ne l'aime.' } \\
\hline
\end{tabular}

\begin{tabular}{|c|c|c|c|c|c|c|c|c|}
\hline c. & $\eta$ & bé & $a$ & fee & $\eta$ & ye & nézesúw-o & sán. \\
\hline & $1 S G$ & COPLOC & $3 S G$ & OBL & $1 \mathrm{SG}$ & SUBJP & vélo-D & acheter \\
\hline & \multicolumn{8}{|c|}{ 'Je veux acheter un vélo.' } \\
\hline
\end{tabular}

\begin{tabular}{|l|l|l|l|l|l|}
\hline$(156)$ & a. & A & síyi & á & kay! \\
\hline & & 3SG & installer & $3 \mathrm{SG}$ & sur \\
\hline & & \multicolumn{3}{|l|}{ 'Pose ça là-dessus!' } \\
\hline
\end{tabular}

\begin{tabular}{|l|l|l|l|l|l|l|}
\hline & b. & Sũy-o & lu & bóyi-ta & á & kay. \\
\hline & & voleur-D & PL & tomber-ACPP & 3SG & sur \\
\hline & \multicolumn{4}{|l|}{ 'Les voleurs l'ont attaqué.' } \\
\hline
\end{tabular}

\begin{tabular}{|l|l|l|l|l|l|l|}
\hline & c. & Domor-óo & be & táabal-óo & kay. & \\
\hline & & nourriture-D & COPLOC & table-D & sur & \\
\hline & & \multicolumn{4}{|l}{ 'La nourriture est sur la table. } \\
\hline
\end{tabular}

\begin{tabular}{|l|l|l|l|l|}
\hline$(157)$ & a. & Tayá & fee & kamma! \\
\hline & & aller & calebasse.D & MTV \\
\hline
\end{tabular}


'Va chercher une calebasse !'

\begin{tabular}{|l|l|l|l|l|l|l|}
\hline & b. & A & wuli-ta & dindiy-o & lú & kamma. \\
\hline & & 3SG & se_lever-ACPP & enfant-D & PL & MTV \\
\hline & & \multicolumn{5}{|c|}{ 'Elle est partie chercher les enfants.' } \\
\hline
\end{tabular}

\begin{tabular}{|l|l|l|l|l|l|l|l|l|l|}
\hline$(158)$ & a. & Nóyolo- $\eta k$-óo & lu & se & séněe & $k e$ & dábǎa & lée & la. \\
\hline & & Niokolo-RESID-D & PL & POTP & cultiver.D & faire & houe.D & FOC & OBL \\
\hline & \multicolumn{7}{|l}{ 'Les gens du Niokolo cultivent à la houe.' } \\
\hline
\end{tabular}

\begin{tabular}{|l|l|l|l|l|l|l|}
\hline & b. & y & náa-ta & wóo & lée & la. \\
\hline & & $1 \mathrm{SG}$ & venir-ACCP & DEM & FOC & ОВL \\
\hline & \multicolumn{5}{|c|}{ 'C'est pour cela que je suis venu.' } \\
\hline
\end{tabular}

\begin{tabular}{|l|l|l|l|l|l|l|}
\hline (159) & a. & A & be & i-te & lée & ma. \\
\hline & & 3SG & COPLOC & 2SG-EMPH & FOC & OBL \\
\hline & \multicolumn{4}{|l}{} \\
\hline
\end{tabular}

\begin{tabular}{|l|l|l|l|l|l|l|}
\hline & b. & Wóo & kaañán-ta & $y$ & ma & lée. \\
\hline & & DEM & être_égal-ACPP & 1SG & OBL & FOC \\
\hline & & \multicolumn{3}{|l}{} \\
\hline
\end{tabular}

\begin{tabular}{|l|l|l|l|l|l|l|l|l|l|}
\hline$(160)$ & a. & Garajkée & lu & sé & $i$ & yélema & túrumáa & ti. & \\
\hline & & Garanké.D & PL & POTP & REFL & transformer & hyène.D & OBL & \\
\hline & \multicolumn{6}{|c|}{ 'Les Garanké peuvent se transformer en hyènes.' } \\
\hline
\end{tabular}

\begin{tabular}{|l|l|l|l|l|l|l|}
\hline & b. & A & kuu-lón-ta & $n$-té & ti & lée. \\
\hline & & $3 S G$ & affaire-savoir-D & 1 SG-EMPH & OBL & FOC \\
\hline & & \multicolumn{4}{|c|}{ 'Il est plus compétent que moi.' } \\
\hline
\end{tabular}




\begin{tabular}{|l|l|l|l|l|l|}
\hline & c. & Álu & náa & ǰỳ-o & ti! \\
\hline & & 2PL & venir & eau-D & OBL \\
\hline & & \multicolumn{3}{|c|}{ 'Apportez de l'eau !' } \\
\hline
\end{tabular}

\begin{tabular}{|l|l|l|l|l|l|}
\hline$(161)$ & a. & J̌y-o & $k e$ & dáyăa & to! \\
\hline & & \multicolumn{4}{|c|}{ 'Mets l'eau dans la jarre!' } \\
\hline & & eau-D & mettre & jarre.D & Loc \\
\hline
\end{tabular}

\begin{tabular}{|l|l|l|l|l|l|l|}
\hline & b. & Mús-ǒo & lu & taða-ta & $k$ õo & to. \\
\hline & & femme-D & PL & aller-ACPP & marigot.D & LOC \\
\hline & \multicolumn{4}{|c|}{ 'Les femmes sont parties au marigot.' } \\
\hline
\end{tabular}

\begin{tabular}{|l|l|l|l|l|l|}
\hline$(162)$ & a. & Wóo & di-yáa-ta & $y$ & yè. \\
\hline & & DEM & être_agréable-ABSTR-ACPP & 1SG & BEN \\
\hline & & 'Ça me plaît.' \\
\hline
\end{tabular}

\begin{tabular}{|l|l|l|l|l|l|l|l|}
\hline & b. & A & ye & básǎa & feenee & luntáy-o & yen. \\
\hline & & 3SG & ACPP & natte.D & étendre & visiteur-D & BEN \\
\hline & & \multicolumn{6}{|c|}{ 'Il a étendu une natte pour le visiteur.' } \\
\hline
\end{tabular}

\begin{tabular}{|l|l|l|l|l|l|l|l|}
\hline & c. & Á & ye & bas-óo & feere & á & yey. \\
\hline & & 3SG & ACPP & remède-D & procurer & 3SG & BEN \\
\hline & \multicolumn{6}{|l}{} \\
\hline
\end{tabular}

\subsection{Les lexèmes nominaux employés comme postpositions}

5 La manifestation la plus évidente de l'emploi de certains lexèmes nominaux comme postpositions est qu'en tant que postpositions, les termes en question sont invariablement dépourvus de la marque de détermination nominale. La liste des lexèmes nominaux aptes à cet emploi peut varier d'une variété de mandingue à l'autre $\mathrm{du}$ fait que les significations qu'ils expriment peuvent souvent s'exprimer aussi par des 
postpositions composées du type présenté en 16.4. Pour le maninka du Niokolo, j'ai relevé les lexèmes nominaux suivants employés en fonction de postposition :

- bulu 'main' $\rightarrow$ 'dans la sphère personnelle de'

\begin{tabular}{|l|l|l|l|l|}
\hline (163) & Godi & te & $y$ & bulu. \\
\hline & argent & COPN & 1 SG & SPHP \\
\hline & 'Je n'ai pas d'argent.' \\
\hline
\end{tabular}

- kono 'ventre' $\rightarrow$ 'dans'

\begin{tabular}{|l|l|l|l|l|l|l|}
\hline$(164)$ & a. & Múy & be & ñí & bóotóo & kono? \\
\hline & & quoi & COPLOC & DEM & sac.D & dans \\
\hline & \multicolumn{4}{|l|}{ 'Qu'y a-t-il dans ce sac?' } \\
\hline
\end{tabular}

\begin{tabular}{|l|l|l|l|l|l|l|l|}
\hline & b. & I & lá & domor-óo & be & fée & kono. \\
\hline & & 2SG & GEN & nourriture-D & COPLOc & calebasse-D & dans \\
\hline & \multicolumn{5}{|c|}{ 'Ton repas est dans la calebasse.' } \\
\hline
\end{tabular}

- koo 'dos' $\rightarrow$ 'en l'absence de, sans'

\begin{tabular}{|c|c|c|c|c|c|c|c|c|c|}
\hline$(165)$ & a. & $M 0$ & meise & $k e$ & fey & $t i i^{-\zeta}$ & $i$ & 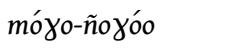 & koo. \\
\hline & & personne.D & POTN & devenir & chose & OBL & $2 S G$ & personne-ASSOC.D & sans \\
\hline & & \multicolumn{8}{|c|}{ 'L'être humain n'est rien sans ses semblables. } \\
\hline
\end{tabular}

\begin{tabular}{|c|c|c|c|c|c|c|c|c|c|c|}
\hline b. & $y$ & na $a-t a$ & $i$ & la & lǔw-o & $m a$, & $y$ & foo-tais & $i$ & koo. \\
\hline & $1 \mathrm{SG}$ & venir-ACPP & $2 S G$ & GEN & maison-D & OBL & $1 \mathrm{SG}$ & manquer-ACPP & $2 S \mathrm{SG}$ & en_l'absence \\
\hline & & & & & t'ai $m$ & & & & & \\
\hline
\end{tabular}

- koto 'dessous, signification' $\rightarrow$ 'sous'

\begin{tabular}{|l|l|l|l|l|l|}
\hline$(166)$ & a. & Siisée & dún-ta & laarán-o & kóto. \\
\hline & & poule.D & entrer-ACPP & lit-D & sous \\
\hline & & \multicolumn{3}{|c|}{ 'La poule s'est glissée sous le lit.' } \\
\hline
\end{tabular}




\begin{tabular}{|l|l|l|l|l|l|}
\hline & b. & Mún & be & bántáy-o & koto? \\
\hline & & quoi & CoPLoc & fromager-D & sous \\
\hline & & \multicolumn{4}{|c|}{ 'Qu'est-ce qu'il y a sous le fromager ?' } \\
\hline
\end{tabular}

- kúy 'tête' $\rightarrow$ 'dans la sphère personnelle de' (par rapport à bulu, apparemment plus fréquent, kuín semble évoquer une relation moins étroite, plus épisodique)

\begin{tabular}{|l|l|l|l|l|l|}
\hline$(167)$ & a. & Godí & te & a & kúy. \\
\hline & & argent & COPN & 1 SG & SPHP \\
\hline & & \multicolumn{4}{|c|}{ 'Il n'a pas d'argent sur lui.' } \\
\hline
\end{tabular}

\begin{tabular}{|l|l|l|l|l|l|l|l|l|}
\hline & b. & A & may & síri & a & kée & kún & foloo. \\
\hline & & $3 S G$ & ACPN & s'installer & $35 \mathrm{SG}$ & mari & SPHP & pour_l'instant \\
\hline & \multicolumn{6}{|c|}{} \\
\hline
\end{tabular}

- kuu 'affaire' $\rightarrow$ 'à propos de'

\begin{tabular}{|c|c|c|c|c|c|c|}
\hline (168) & a. & 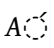 & yili-buíla-ta & $a$ & $n a$ & kuu. \\
\hline & & $3 S G$ & esprit-laisser-ACPP & $3 S G$ & mère.D & à_propos \\
\hline & & \multicolumn{5}{|c|}{ 'Il s'est souvenu de sa mère.' } \\
\hline
\end{tabular}

\begin{tabular}{|c|c|c|c|c|}
\hline b. & Kana & 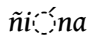 & $y$ & kuu! \\
\hline & SUBJN & oublier & $1 \mathrm{PL}$ & à_propos \\
\hline & \multicolumn{4}{|c|}{ 'Ne nous oublie pas!' } \\
\hline
\end{tabular}

- ñaa 'œil' $\rightarrow$ 'devant, avant'

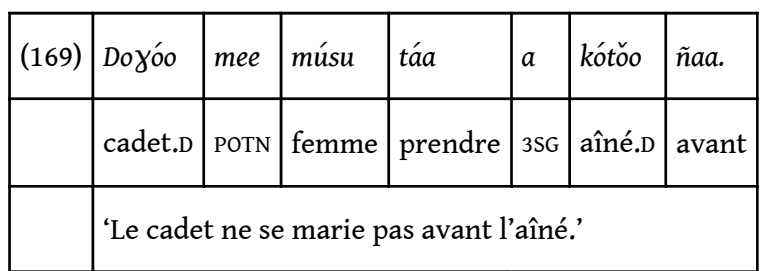

- tee 'taille (milieu du corps)' $\rightarrow$ 'entre' 


\begin{tabular}{|l|l|l|l|l|l|}
\hline$(170)$ & a. & Múy & be & álu & tee? \\
\hline & & quoi & COPLOC & 2PL & entre \\
\hline & & \multicolumn{3}{|c|}{ 'Qu'y a-t-il entre vous?’ } \\
\hline
\end{tabular}

\begin{tabular}{|c|c|c|c|c|c|c|c|c|c|c|c|}
\hline b. & Wo & sain-o & nin & ñinay & tee, & $a ́$ & ye & sañjjii & wooró & bo & lée. \\
\hline & DEM & année-D & avec & année_en_cours & entre & $3 S G$ & ACPP & année & six & sortir & FOC \\
\hline & 'Entr & & & & & & & & & & \\
\hline
\end{tabular}

- yáa 'demeure' $\rightarrow$ 'chez'

\begin{tabular}{|c|c|c|c|c|c|}
\hline (171) & $\eta$ & baabá & be & 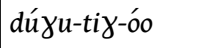 & yáa. \\
\hline & $1 S G$ & papa & COPLOC & village-maître-D & chez \\
\hline & \multicolumn{5}{|c|}{ 'Mon père est chez le chef du village. } \\
\hline
\end{tabular}

\subsection{Postpositions composées}

6 Le maninka a un inventaire important de postpositions composées formées par la juxtaposition d'un nom à la forme nue et de l'une des postpositions énumérées en 16.2. Beaucoup de ces composés ont aussi un emploi adverbial, c'est-à-dire sans le complément qui les précède dans leur emploi comme postpositions.

7 La liste donnée ici ne prétend pas à l'exhaustivité. On ne peut en outre pas exclure la possibilité de créations occasionnelles, comme da'-ibala 'à l'épaule de', attesté dans un proverbe.

- daafee 'à côté de' (cf. daa 'bord, ouverture, endroit')

\begin{tabular}{|l|l|l|l|l|}
\hline (172) & A & síyi-ta & kéebáa & daafee. \\
\hline & 3SG & s'asseoir-ACPP & vieux.D & à_côté \\
\hline & \multicolumn{3}{|l}{ 'Il s'est assis à côté du vieux.' } \\
\hline
\end{tabular}

- daala 'au bord de' (cf. daa 'bord, ouverture, endroit')

\begin{tabular}{|l|l|l|l|l|l|l|}
\hline$(173)$ & ń & na & sáatée & be & băa & daala. \\
\hline & 1 PL & GEN & village.D & COPLOc & fleuve.D & au_bord \\
\hline
\end{tabular}


'Notre village est au bord du fleuve.'

- koola 'après' (cf. koo 'dos')

\begin{tabular}{|l|l|l|l|l|l|l|}
\hline$(174)$ & $\eta$ & yá & $\eta$ & domo-ri & $a ́$ & koola. \\
\hline & 1 SG & ACPP & REFL & manger-ANTIP & 3SG & après \\
\hline \multicolumn{6}{|l}{} \\
\hline
\end{tabular}

- kooma 'derrière’ (cf. koo ‘dos’)

\begin{tabular}{|l|l|l|l|l|l|}
\hline$(175)$ & $A$ & taýá-ta & sǔw-o & kooma & lée. \\
\hline & 3SG & partir-ACPP & maison-D & derrière & FOC \\
\hline \multicolumn{6}{|c}{} \\
\hline
\end{tabular}

- kúnna 'sur, au-dessus' (cf. kúy 'tête’)

\begin{tabular}{|l|l|l|l|l|}
\hline (176) & Fázan-óo & feenee & feee & kúnna! \\
\hline & pagne-D & étendre & calebasse.D & au_dessus \\
\hline & \multicolumn{3}{|l}{ 'Etends le pagne sur la calebasse!' } \\
\hline
\end{tabular}

• kúnto ‘au sommet, au-dessus' (cf. kúy ‘tête')

\begin{tabular}{|l|l|l|l|l|l|}
\hline (177) & $\tilde{N}$ ì & née & be & $\eta$ & kúnto. \\
\hline & DEM & FOC & COPLOC & 1 SG & au_dessus \\
\hline & 'C'est ce qui me préoccupe.' \\
\hline
\end{tabular}

- máafanna 'du côté de’ (cf. máafay ‘côté’)

\begin{tabular}{|l|l|l|l|l|l|l|l|}
\hline$(178)$ & I & lá & domor-óo & fele & síyi-rin & dăa & máafanna. \\
\hline & 2SG & GEN & nourriture-D & voici & installer-D & porte.D & du_côté \\
\hline \multicolumn{7}{|l}{} \\
\hline
\end{tabular}

- ñaala 'en présence de’ (cf. ñaa'œil')

\begin{tabular}{|l|l|l|l|l|l|}
\hline$(179)$ & $\tilde{N} i \eta$ & $\tilde{n} a ́ \gamma \check{a}-\eta$ & $k e-t a ́$ & $\eta$ & $\tilde{n} a a l a$. \\
\hline
\end{tabular}




\begin{tabular}{|l|l|l|l|l|l|}
\hline & DEM & fête-DEF & avoir_lieu-ACPP & 1SG & en_présence \\
\hline & 'Cette cérémonie a eu lieu en ma présence.' \\
\hline
\end{tabular}

- ñaato 'devant' (cf. ñaa 'œil')

\begin{tabular}{|l|l|l|l|}
\hline$(180)$ & Támbi & $\eta$ & ñaato! \\
\hline & passer & 1 SSG & devant \\
\hline & 'Passe devant moi !' & & \\
\hline
\end{tabular}

- sanna, santo 'en haut' (cf. say 'ciel')

\begin{tabular}{|l|l|l|l|l|l|}
\hline$(181)$ & a. & Foñimáa & yéle-ta & bántán-o & sanna. \\
\hline & & singe.D & grimper-ACPP & fromager-D & en_haut \\
\hline & & 'Le singe a grimpé au sommet du fromager.' \\
\hline
\end{tabular}

\begin{tabular}{|l|l|l|l|l|l|l|l|}
\hline & b. & N-té-́ & la & kenáa & fele & kónko-ríy-o & santo. \\
\hline & & 1 SG-EMPH & GEN & champ.D & voici & colline-DIM-D & en_haut \\
\hline & \multicolumn{3}{|c}{ 'Mon champ est en haut de la colline.' } \\
\hline
\end{tabular}

• sínna 'à côté de, près de' (cf. síy 'pied, jambe')

\begin{tabular}{|l|l|l|l|l|}
\hline (182) & Yendée & la-ndì & dábǎa & sínna! \\
\hline & hache.D & s'étendre-CAus & houe.D & à_côté \\
\hline & \multicolumn{3}{|l}{ 'Pose la hache à côté de la houe!' } \\
\hline
\end{tabular}

- teema 'au milieu' (cf. tee 'milieu du corps')

\begin{tabular}{|l|l|l|l|l|l|l|l|}
\hline (183) & a. & A & ye & jálǎa & búla & bǎa & teema. \\
\hline & & 3SG & ACPP & filet.D & lancer & fleuve.D & au_milieu \\
\hline & \multicolumn{6}{|c|}{ 'Il a lancé le filet au milieu du fleuve.' } \\
\hline
\end{tabular}

\begin{tabular}{|l|l|l|l|l|l|l|l|}
\hline & b. & A & ye & bǔ̀-o & lóo & sáatée & teema. \\
\hline & & $35 \mathrm{SG}$ & ACPP & case-D & construire & village.D & au_milieu \\
\hline
\end{tabular}


'Il a construit une case au milieu du village.'

\subsection{Prépositions}

- Diina 'au lieu de, plutôt que' prend comme complément un groupe nominal marqué de la postposition ti (qui peut à elle seule exprimer un sens de comparaison).

\begin{tabular}{|l|l|l|l|l|l|l|l|l|}
\hline$(184)$ & a. & I & $\tilde{n}$ ńn-ta & baar-óo & ke-lá & diiná & tulúy-o & ti. \\
\hline & & 2SG & devoir-D & travail-D & faire-INF & plutôt_que & jeu-D & OBL \\
\hline & \multicolumn{7}{|l}{} \\
\hline
\end{tabular}

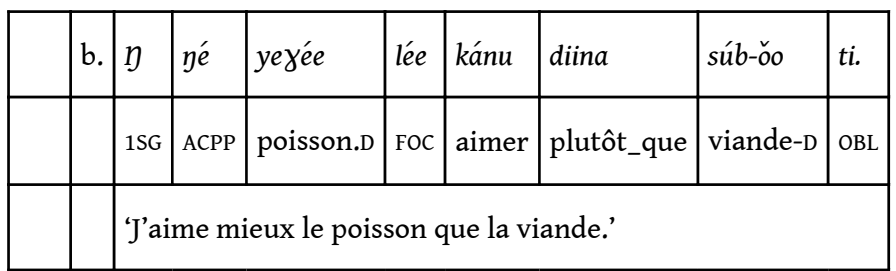

- Fo 'jusqu'à' prend comme complément, soit un groupe postpositionnel, soit un groupe nominal apte à fonction comme complément de lieu ou de temps sans l'adjonction d'une postposition.

\begin{tabular}{|l|l|l|l|l|l|l|l|l|l|}
\hline$(185)$ & $A$ & tu-tá & $\eta$ & $n a ́$ & lǔw-o & $m a$ & fo & fitir-óo & $m a$. \\
\hline & $3 \mathrm{SG}$ & rester-ACPP & $1 \mathrm{SG}$ & GEN & maison-D & OBL & jusqu'à & crépuscule-D & OBL \\
\hline \multicolumn{7}{|c|}{} \\
\hline
\end{tabular}

- Hake 'à cause de' prend comme complément un constituant nominal.

\begin{tabular}{|c|c|c|c|c|c|c|c|c|}
\hline$(186)$ & $\eta$ & $y a^{i-5}$ & $a$ & $k a^{i-i n n}$ & hake & $a a^{i-5}$ & la & $\tilde{n} i_{-}^{-} i n a^{-}$ \\
\hline & $1 S G$ & ACPP & $3 S G$ & aimer & à_cause_de & $3 S G$ & GEN & gentillesse.D \\
\hline & \multicolumn{8}{|c|}{ 'Je l'aime à cause de sa gentillesse.' } \\
\hline
\end{tabular}

- Kábiriy 'depuis' prend comme complément, soit un groupe postpositionnel, soit un groupe nominal apte à fonctionner comme complément de lieu ou de temps sans l'adjonction d'une postposition.

\begin{tabular}{|l|l|l|l|l|l|l|}
\hline (187) & Kábirí & seruy & $a ́$ & be & kúura_́n-din & née. \\
\hline & depuis & l'an_dernier & 3SG & COPLOC & tombrt_malade-RES & FOC \\
\hline
\end{tabular}


'Il est malade depuis l'an dernier.'

- Kómu et (i) ko 'comme' s'utilisent de façon semblable, mais (i) ko, (étymologiquement 'on dirait', avec un pronom de 2ème personne en valeur générique) résulte de la grammaticalisation du quotatif ko (cf. 22.2), tandis que ko

\begin{tabular}{|c|c|c|c|c|c|c|c|c|c|}
\hline \multirow[t]{2}{*}{ (188) } & a. & Dindi & lu & $b e e^{i-5}$ & tulu-n-na & (i) & ko & $b \tilde{a} a$ & lu. \\
\hline & & enfant-D & PL & COPLOC & jouer-INF & $2 S G$ & QUOT & chèvre.D & PL \\
\hline & & 0 & & & & & & & \\
\hline
\end{tabular}

\begin{tabular}{|c|c|c|c|c|c|c|c|c|c|c|}
\hline b. & Ko:-́mu & ke-́e-kundaía, & mus-o & $l u$ & fanay & $s e-e_{0}^{\prime}$ & nam-o & lu & $k e$ & leíe. \\
\hline & comme & homme-chez.D & femme-D & $\mathrm{PL}$ & aussi & РОТР & rite-D & PL & faire & FOC \\
\hline & Con & & & & & & le rito & & & \\
\hline
\end{tabular}

- Pur (du français 'pour') s'utilise de manière redondante avec la postposition bénéfactive yey.

\subsection{Infinitifs grammaticalisés comme prépositions}

Deux cas d'infinitifs grammaticalisés comme postpositions ont été relevés : ka 'depuis' (< táa 'prendre) et ká siyaa (< siyaa 'être abondant'), qui en prenant comme complément un groupe nominal marqué de la postposition ti exprime 'plus que'.

\begin{tabular}{|l|l|l|l|l|l|l|l|l|l|l|l|l|l|}
\hline (189) & a. & Ká & a & táa & jáy & $m a$ & fo & jée, & kilo & bii & sába & lée & mu. \\
\hline & & INF & 3 SG & prendre & ici & OBL & jusqu'à & là & kilomètre & dix & trois & FOC & COPID \\
\hline \multicolumn{10}{|c|}{ D'ici jusque là, ça fait trente kilomètres.' } \\
\hline
\end{tabular}

\begin{tabular}{|l|l|l|l|l|l|l|l|l|l|}
\hline & b. & A & ye & $i$ & lon & ká & siyáa & $n$-té & ti. \\
\hline & & 3SG & ACPP & 2SG & connaître & INF & être_abondant & 1SG-EMPH & OBL \\
\hline & \multicolumn{6}{|c|}{} \\
\hline
\end{tabular}




\section{AUTEUR}

DENIS CREISSELS

Université de Lyon

Denis.Creissels@univ-lyon2.fr 